\title{
Drug Induced Psychosis
}

\author{
ROB POOLE and CLARE BRABBINS
}

Differential diagnosis of psychotic disorder in the young routinely includes "? Drug induced psychosis". Hitherto this diagnostic category has had no consistent definition and the relationship between drug use and psychotic symptoms is controversial. Drug use is a major complicating factor in psychosis, renders the management of psychotic disorders more difficult, and adverse reactions to recreational drugs may mimic psychosis. We have collected data suggesting that psychiatrists of all grades lack clarity in their understanding of the relationship between psychosis and drug use (Brabbins \& Poole, in preparation). The confusion may have its origin in personal experience; the sub-culture of drug use is alien to most psychiatrists, who rarely have had first-hand experience of it. The sub-culture of alcohol abuse, on the other hand, is more apposite and familiar. There is no such confusion about the adverse effects of this.

The consequences of mis-diagnosis are serious, whether a functional psychosis is mistaken for a drug reaction or vice versa. The inquiry into the case of Christopher Clunis (Ritchie et al, 1994) drew attention to a number of failings in good practice as a consequence of which a man with schizophrenia did not receive satisfactory treatment, and went on to kill. Among the errors identified was the uncritical attribution of his psychotic symptoms to drug use. Although such tragedies are rare, the diagnostic error is common.

In order to understand the various abnormal mental states related to drug use a rational and generally accepted framework is necessary, but there are several obstructions to clarity. Patterns of drug taking alter rapidly with changing fashions, drug availability and shifting sub-cultural values (Wright \& Pearl, 1995). Drugs which were virtually unknown in British society until recently, such as free base cocaine (crack) and 3,4-methylenedioxymethamphetamine (ecstasy), are now widely available. There are major methodological problems in teasing out the various causal relationships (Thornicroft, 1990), and the literature as a whole is marked by the inference of causal relationships which are not supported by the data they are based upon. With a few notable exceptions, existing published studies of psychotic subjects who have taken drugs are largely uncontrolled, lack data on course and outcome, and rarely relate mental state to toxicological findings. Experimental studies convincingly demonstrate that a number of drugs can produce transient psychotic symptoms in some subjects when intoxicated (Isbell et al, 1967; Angrist \& Gershon, 1970) and exacerbate existing psychotic symptoms (Janowsky et al, 1973; Negrete et al, 1986) but these studies cannot explore drug use as it occurs in the real world.

ICD-10 (World Health Organization, 1992) is disappointing in its approach to drug-related states. The classification used is vague, inconclusive, and does not address the variety of potential interactions between drugs and psychotic states. The recently published DSM-IV (American Psychiatric Association, 1994) is better in-so-far as it provides a classification of some drug reactions by substance, but the approach is too pedantic to relate easily to common clinical situations and it does not take into account poly-drug use. There is no published research on the management of drug-related psychotic states nor non-dependent drug misuse, and advice to desist from drug use is generally ineffective.

The following classification of potential interactions between psychosis and drug use is based upon the extensive but flawed literature. Given the poor scientific status of the supporting data, it is provisional and requires evaluation. It is intended to introduce clarity into a dangerously confused area.

\section{Intoxication mimicking functional psychosis}

This refers to a direct pharmacological effect. There is no doubt that this occurs both with stimulants (Connell, 1958; Bell, 1965; Satel et al, 1991) and cannabis (Mathers \& Ghodse, 1992). It probably also occurs with solvents, ecstasy and lysergic acid diethylamide (LSD). Such states may persist for several days; it is not commonly appreciated that some of these substances have extremely long halflives. For example, amphetamine may persist in measurable quantities in the urine for up to 48 hours after a single small dose, and cannabinoids, even excluding active metabolites, persist in measurable quantities for up to 6 weeks. 


\section{Pathoplastic reactions in functional psychosis}

There is a prima facie case that psychoactive drugs substantially alter the clinical picture in functional psychoses, and indeed there is some evidence that this is the case, for example schizophreniform symptoms appearing transiently in affective disorder after cannabis use (Harding \& Knight, 1973) and schizophrenic symptoms worsening after stimulant use (Janowsky et al, 1973). An increased level of disturbed behaviour and violence under these circumstances is to be expected (Swanson et al, 1990), and management is more difficult (Knudsen \& Vilmar, 1984).

\section{Chronic hallucinosis induced by substance abuse}

This includes alcoholic hallucinosis (Lishman, 1987), LSD flashbacks (Abraham, 1983) and cannabis flashbacks (Tunving, 1985). The features of these states are not identical, but they have in common the fact that they can continue through long periods of abstinence. The mechanisms by which such states occur are obscure. Flashbacks (recurrence of the features of drug intoxication after cessation of drug use) are true hallucinations, having the quality of true perceptions. While they are often frightening, the subject recognises that they arise from within themselves, and frequently attributes them to the previous drug use. If this quality of insight is absent, such a diagnosis is in doubt. Alcoholic hallucinosis occurs in clear consciousness and usually consists of vivid auditory hallucinations. There are no other symptoms of psychosis and any false beliefs are transient and secondary to the hallucinatory experiences.

\section{Drug induced relapse of functional psychosis}

Drug use, particularly of stimulants and cannabis, is said to be a potent precipitant of relapse of an existing psychotic illness (Bell, 1965; Breakey et al, 1974; Treffert, 1978). The question of causality is more complicated than it may at first appear. There are three major possibilities:

(a) Patients may try to 'treat' themselves with illegal drugs during the early stages of psychotic relapse, the drug use therefore being a symptom rather than a cause of illness

(b) Patients may be more likely to use drugs when ill as a consequence of impaired judgement

(c) Intoxication itself may directly provoke relapse.

Which of the above pertains is an important question which remains unresolved through the naïve assumption that causality self-evidently flows from drug use to relapse rather than vice versa.

\section{Withdrawal states}

The major syndrome is delirium tremens, normally associated with alcohol withdrawal, but also reported with barbiturate or benzodiazepine withdrawal (Lishman, 1987). These states have features distinct from the major functional psychoses, such as clouding of consciousness and vivid visual hallucinations. Difficulty in making the diagnosis arises usually when the relevant substance misuse history has not been or cannot be obtained, particularly with the rarer syndromes of barbiturate and benzodiazepine delirium tremens. The presence of clouding of consciousness and complex visual hallucinations, while not pathognomonic, raises the possibility of a withdrawal state in the absence of other organic factors.

\section{Other reactions}

There is a group of psychological reactions to drug use which can rarely mimic psychosis.

\section{Intoxication with clouding of consciousness}

Acute confusional states may arise during intoxication with a range of drugs, although there is not a close resemblance to functional psychosis. Features include fragmentary hallucinosis, paranoid ideas, agitation, clouding of consciousness and marked fluctuation of symptoms over the 24-hour period.

\section{Post intoxication depression}

Stimulants cause a marked "crash", correctly described as post-intoxication depression (Gawin \& Kleber, 1986; Lowenstein et al, 1987). While this does not resemble psychosis, it may be so severe as to induce a suicidal crisis.

\section{Panic attacks}

Hallucinogens, including cannabis (Ames, 1958; Kotin et al, 1973) and LSD can induce severe panic. The majority of patients report having a bad trip but the combination of extreme anxiety with hallucinations may be mistaken for functional psychosis.

\section{True drug induced psychosis}

If the term 'drug induced psychosis' has any utility it refers to psychotic symptoms which arise in the context of drug intoxication but persist beyond elimination of the drug. It only recurs if a subject is re-exposed to the drug, and it may be an idiosyncratic or dose-dependent syndrome. If drug induced psychosis is to qualify as a separate entity, it must have a different course and outcome to the 
major functional psychoses. Many scientific papers assert that the existence of such a syndrome is established with respect to amphetamines, and perhaps cocaine (Gold \& Bowers, 1978; McGuire \& Fahy, 1991; Hassiotis \& Taylor, 1992). This is commonly taught to psychiatrists in training and DSM-IV refers to such a syndrome. Those papers claiming to demonstrate the existence of such a syndrome do not present data supporting this conclusion (e.g. McGuire et al, 1994; Poole \& Brabbins, 1994). Many papers cite as evidence either Connell (1958) or Bell (1965). In fact neither Connell's nor Bell's work described such a syndrome and Connell has recently confirmed (personal communication, 1994) that he has been widely misquoted; he believes that he demonstrated that psychotic symptoms precipitated by amphetamines only occurred in relation to intoxication, measured biochemically by urine tests.

Only one study has presented evidence for the existence of such a syndrome. A historical prospective study (Andreason et al, 1987) has shown an association between self-report of heavy cannabis use on conscription to the Swedish armed forces and later admission for schizophrenia. The method has been criticised on several counts (Negrete, 1989) and the authors neglected confounding variables independently associated with early cannabis use on the one hand, and later psychotic illness on the other. Moreover, the assertion was of a causal link between early cannabis use and the development of schizophrenia per se, rather than a clearly delineated 'drug induced psychosis' syndrome as defined above.

\section{Causality}

The common clinical and scientific error is to uncritically assume that drug use, as an organic factor, is causal rather than symptomatic. This view has its origin in the hierarchical approach to diagnosis in psychiatry, and some authorities go as far as to suggest that a diagnosis of major psychosis should not be made in the presence of drug use. DSM-IV takes the converse position that a functional diagnosis should not be excluded unless there is compelling clinical evidence that the symptoms are entirely attributable to drugs. The latter position is to be preferred, as it is both in keeping with the limited scientific evidence and mitigates for clinical safety. If the symptoms are persistent and the individual cannot be persuaded to discontinue drug use, then causation may be irrelevant. Drawing a distinction between such patients and those with "real" mental illness is ethically questionable. Both types of patients suffer psychological distress and it is not the physician's role to moralise. It is important to recognise that, among the large group of drug users within the general population, a proportion will become mentally ill regardless of any supposed psychotomimetic properties of drugs.

\section{Future research}

The nature and management of drug induced abnormal mental states can only be clarified by better quality studies. Authors should not make assertions that are unsupported by their data; speculation should be identified as such. There is a plethora of single case reports and short uncontrolled series which add very little to knowledge and are best avoided. There is a need for a large scale naturalistic study comparing the long-term outcome of psychotic patients who have a history of drug use with other psychotic patients. Such a study would need to incorporate toxicological data and pay particular attention to confounding variables, such as poor premorbid adjustment, which are associated with both drug abuse and poor outcome in major mental illness. In the face of conflicting evidence as to whether neuroleptics are effective in controlling psychotic symptoms related to drug use, there is a need for studies of treatment strategies, both pharmacological and psychosocial (Smith \& Hucker, 1994). Any credible description of true 'drug induced psychosis' as defined above should be reported.

\section{Conclusions}

The above coherent, empirical (though unproven) classification of adverse reactions to drug use offers the opportunity to discontinue use of the term 'drug induced psychosis', which is ambiguous and unsustainable. Other similarly ambiguous clinical terms have proven difficult to eradicate (e.g. "hysteria" and "formulation"). However, confused terminology leads to confused management, and the unrestrained spread of the drug epidemic demands the adoption of more rigorous thinking.

\section{References}

Abraham, H. D. (1983) Visual phenomenology of the LSD flashback. Archives of General Psychiatry, 40, 884-889.

American Psychiatric Association (1994) Diagnostic and Statistical Manual of Mental Disorders (4th edn) (DSM-IV). Washington, DC: APA.

AMEs, F. (1958) A clinical and metabolic study of acute intoxication with Cannabis sativa and its role in the model psychoses. Journal of Mental Science, 104, 972-999.

Andreason, S., Allebeck, P., Enastrom, A., et al (1987) Cannabis and schizophrenia. A longitudinal study of Swedish conscripts. Lancet, ii, 1483-1486. 
Angrist, B. M. \& Gershon, S. (1970) The phenomenology of experimentally induced amphetamine psychosis: Preliminary observations. Biological Psychiatry, 2, 95-107.

Bt:LI, D. S. (1965) Comparison of amphetamine psychosis and schizophrenia. British Journal of Psychiatry, 111, 701-707.

Breakey, W. R., Goodell, H., Lorenz, P. C., et al (1974) Hallucinogenic drugs as precipitants of schizophrenia Psychological Medicine, 4, 255-261.

COHFN, S. I. \& JOHNSON, K. (1988) Psychosis from alcohol or drug abuse. British Medical Journal, 297, 1270-1271.

Connel. . P. H. (1958) Amphetamine Psychosis. Institute of Psychiatry Maudsley Monograph No. 5. London: Oxford University Press.

GAwin, F. H. \& KLEBER, H. D. (1986) Abstinence symptomatology and psychiatric diagnosis in cocaine abusers. Clinical observations. Archives of General Psychiatry, 43, 107-113.

Got.D, M. S. \& Bowers, M. B. (1978) Neurobiological vulnerability to low dose amphetamine psychosis. American Journal of Psychiatry, 135, 1546-1548.

HaRding, T. \& KNIGHT, F. (1973) Marijuana modified mania. Archives of General Psychiatry, 29, 635-637.

Hassiotis, A. \& TAYlor, J. (1992) Psychotic illness following 'Mabi Bark Tea' consumption. British Journal of Psychiatry, 161, 404-407.

IsBel., C., Gorodetzsky, C., JASINSKI, D., et al (1967) Effects of Delta-9-transtetrahydrocannabinol in man. Psychopharmacologia. 11, 691-696

Janowsky, D. S., El-Yousef, M. K., Davis, J. M., et al (1973) Provocation of schizophrenic symptoms by intravenous administration of methylphenidate. Archives of General Psychiatry, 28, 185-191.

KnUdesen, P. \& VILMAR, T. (1984) Cannabis and neuroleptic agents in schizophrenia. Acta Psychiatrica Scandinavica, 69, 162-174.

Kotin, J., Post, R. M. \& Goodwin, K. (1973) Delta-9-tetrahydrocannabinol in depressed patients. Archives of General Psychiatry, 28, 345-348.

Lowenstein, D. H., Massa, S. M., Rowbotham, M. C., et al (1987) Acute neurological and psychiatric complications associated with cocaine abuse. American Journal of Medicine, 83, $841-846$.

Lishman, W. A. (1987) Organic Psychiatry. The Psychological
Consequences of Cerebral Disorder. Oxford: Blackwell Scientific Publications.

Mathers, D. C. \& Ghodse, A. H. (1992) Cannabis and psychotic illness. British Journal of Psychiatry, 161, 648-653.

MCGUIRE, P. \& FAHY, T. (1991) Chronic paranoid psychosis after misuse of MDMA (ecstasy). British Medical Journal, 302, 697. - COPE, H. \& FAHY, T. A. (1994) Diversity of psychopathology associated with use of 3,4-Methylenedioxymethamphetamine ('Ecstasy'). British Journal of Psychiatry, 165, 391-395.

NEGRETE, J. C. (1989) Cannabis and schizophrenia. British Journal of Addiction, 84, 349-351.

, Knapp, W. P., Douglas, D. E., et al (1986) Cannabis affects the severity of schizophrenic symptoms: results of a clinical survey. Psychological Medicine, 16, 515-520.

PoOle, R. G. \& BrabBins, C. J. (1994) Psychopathology and 'ecstasy'. British Journal of Psychiatry, 165, 837.

Ritchie, J., Dick, D. \& Lingham, R. (1994) The Report of the Inquiry into the Care and Treatment of Christopher Clunis. London: HMSO.

Satel, S. L., Southwick, S. M. \& Gawin, F. H. (1991) Clinical features of cocaine induced paranoia. American Journal of Psychiatry, 148, 495-498.

SMIth, J. \& Hucker, S. (1994) Schizophrenia and substance abuse. British Journal of Psychiatry, 165, 13-21.

Swanson, J. W., Holzer, C. E., Ganju, V. K., et al (1990) Violence and psychiatric disorder in the community: Evidence from the epidemiologic catchment area surveys. Hospital and Community Psychiatry, 41, 761-770.

ThORNicroft, G. (1990) Cannabis and psychosis. Is there epidemiological evidence for an association? British Journal of Psychiatry, 157, 25-33.

TrefFerT, D. A. (1978) Marijuana use in schizophrenia: A clear hazard. American Journal of Psychiatry, 135, 1213-1215.

Tunving, K. (1985) Psychiatric effects of cannabis use. Acta Psychiatrica Scandinavica, 72, 209-217.

World Health Organization (1992) The Tenth Revision of the International Classification of Diseases and Related Health Problems (ICD-10). Geneva: WHO.

Wright, J. D. \& PearL, L. (1995) Knowledge and experience of young people regarding drug misuse, 1969-94. British Medical Journal, 310, 20-24.

Rob Poole, MRCPsych, Clare Brabbins, MRCPsych, Windsor House, Community Mental Health Resource Centre, Liverpool

Correspondence: Dr R. Poole, Windsor House, Community Mental Health Resource Centre, 40, Upper Parliament Street, Liverpool, L8 7LF

(First received II May 1995, final revision 26 July 1995, accepted 18 August 1995) 